\title{
LINKED AND UNSTABLE RESISTANCE TO KANAMYCIN AND PENICILLIN, AND DIFFUSIBLE PIGMENT PRODUCTION, IN AN ISOLATE OF STAPHYLOCOCCUS AUREUS
}

\author{
D. I. Annear and W. B. Grubb \\ Department of Microbiology, Royal Perth Hospital, and Department of Microbiology, \\ The University of Western Australia
}

\section{Plate VII}

UNSTABLE penicillin resistance in Staphylococcus aureus was first studied by Barber (1949). Subsequent work on this subject has been reviewed by Novick (1967) and Richmond (1968). Penicillinase production is often controlled by an extrachromosomal genetic element (plasmid) and resistance to mercury (Richmond and John, 1964); other inorganic salts (Novick and Roth, 1968) and erythromycin (Mitsuhashi et al., 1965) may be controlled by the same plasmid. In addition, Evans and Waterworth (1966) have reported a possible linkage of resistance to penicillin and fusidic acid.

Linkage of kanamycin and penicillin resistance has now been seen in a strain of Staph. aureus (no. 5464) recently isolated from an infected wound in this hospital. It was demonstrated both in organisms grown directly from the wound and in the progeny from these cells. The organism proved to be of additional interest in that it produces an intense yellow diffusible pigment apparently identical with that previously seen in a methicillin-resistant isolate of Staph. aureus (Annear and Grubb, 1969).

\section{MATERIALS AND METHODS}

Colonies were screened for resistance to antibiotics by multipoint replication (May and Houghton, 1965). Two organisms were always included as controls, one resistant to the antibiotics being tested and the other sensitive. The base medium was Oxoid Blood Agar Base no. 2, and for the two antibiotics of main interest, kanamycin and penicillin, the screening levels were 10 and $1 \mu \mathrm{g}$ per $\mathrm{ml}$ respectively.

Colonies were picked from plates with heat-sterilised wooden toothpicks. The growth was emulsified in three-drop volumes of broth in the wells of Perspex trays and these suspensions were replicated on to agar plates.

Areas of growth that appeared on the plate after incubation will be referred to as " spots". The terms " resistant" and "sensitive" will be used to signify whether the spot did or did not appear on an antibiotic plate. When spots were selected for further work it will be specified whether subculture was made from the control or from the antibiotic plate. Illustrations of typical plates are shown in the figure.

The method of Novick and Richmond (1965) was used to test for penicillinase production.

Testing for pigment production was done as in the earlier study of this phenomenon (Annear and Grubb).

Received 8 Sept. 1971; accepted 20 Sept. 1971.

J. MED. MICROBIOL.-VOL. 5 (1972)

109 


\section{RESULTS}

One hundred colonies from a direct plating from a swab of the lesion were screened for resistance to penicillin and kanamycin. Of the number examined, 98 were resistant to both antibiotics and two were sensitive to them.

A resistant spot was selected from the control plate and subcultured to an agar slope. After several days at room temperature the growth was spread for isolated colonies, which were then screened for resistance. An appreciable fraction ( 24 per cent.) of the spots were sensitive to both kanamycin and penicillin and the remainder were resistant to both. This procedure was carried out three times by serial selection of resistant spots from the control plates. In each screening there was consistent linkage between kanamycin and penicillin. The percentage of sensitive spots in four successive screenings of 50 colonies was $24,42,40$ and 32 . The results of one such screening may be seen in the figure. Screening for resistance to other antibiotics was done in parallel with kanamycin and penicillin and colonies were found to be uniformly resistant to tetracycline and streptomycin and uniformly sensitive to methicillin and lincomycin. The results for erythromycin were equivocal and need further investigation.

Tests for penicillinase on the variants revealed a positive correlation between penicillin resistance and production of the enzyme.

The last batch of 50 colonies was tested and all were found to produce an intense diffusible yellow pigment.

Phage typing of resistant and sensitive variants both directly from the wound and from subsequent cultures showed them all to have the phage-typing pattern $83 \mathrm{~A} / 84 / 85$.

\section{DisCUSSION}

The organisms investigated here when maintained in culture yielded sensitive variants spontaneously and with high frequency, and provide a clear example of unstable and linked resistance to penicillin and kanamycin. Although more exhaustive screening may reveal exceptions, it is obvious that this linkage is very firm. The results strongly suggest that resistance to the two drugs is extrachromosomal and controlled by the same plasmid.

The high frequency with which this organism produces sensitive variants is in contrast with the findings of Mitsuhashi et al. (1965) for linked erythromycin and penicillin resistance. Only one of the strains they investigated produced sensitive variants spontaneously and this was at a frequency of 0.2 per cent. Even after acriflavine treatment, the highest frequency they observed was only $3 \cdot 5$ per cent.

The only other report of antibiotic resistance linked to penicillin resistance is that of fusidic acid reported by Evans and Waterworth (1966). They described this resistance as very unstable but gave no quantitative data.

In the specimen taken directly from the wound, the frequency of sensitive variants was relatively low and they might well have been undetected if fewer colonies had been screened. Little work has yet been done on the measurement of instability of antibiotic resistance as it occurs in vivo in Staph. aureus. 
LiNKED ANTIBIOTIC RESISTANCE IN STAPH. AUREUS

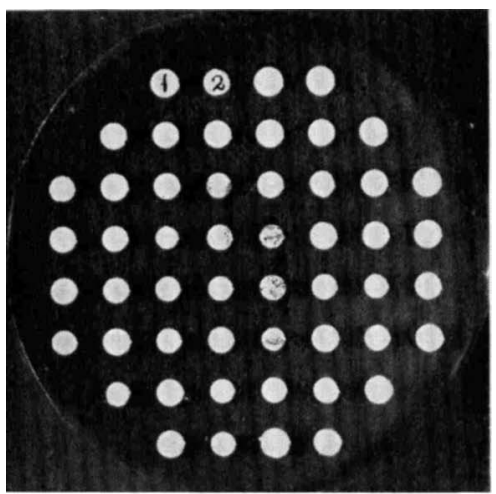

CONTROL

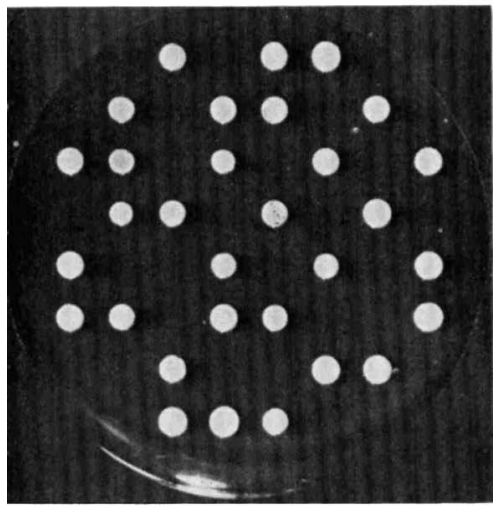

KANAMYCIN

$10 \mu \mathrm{g}$ per $\mathrm{ml}$.

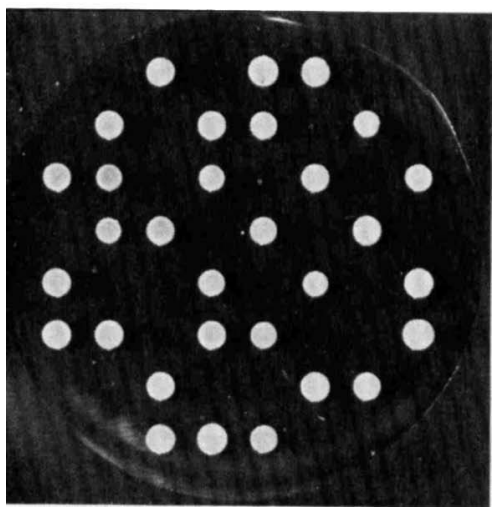

$$
\text { PENICILLIN }
$$

$1 \mu \mathrm{g}$ per $\mathrm{ml}$.

FIgURE.-Antibiotic screening of colonies of Staphylococcus aureus (strain no. 5464), showing consistent linkage between resistance to kanamycin and penicillin. Spots 1 and 2 are respectively resistant and sensitive control strains. 
In our previous work on isolates of Staph. aureus with unstable resistance to kanamycin, we found diffusible pigment production only in the resistant variants (Annear and Grubb, 1969). In the present study, pigment is produced regardless of whether the variants are resistant or sensitive to penicillin and kanamycin. Many other strains of Staph. aureus isolated in this laboratory have been shown to be producers of the diffusible pigment, and further work is needed to determine its nature, the mechanism of its production and its relation, if any, to antibiotic resistance.

\section{SUMMARY}

A strain of Staph. aureus isolated from an infected wound showed firmly linked resistance to penicillin and kanamycin. Variants sensitive to both these antibiotics were produced spontaneously and at a high frequency during storage for a few days at ambient temperature.

Both sensitive and resistant variants produced a highly coloured diffusible pigment.

We wish to thank Dr E. M. Mackay-Scollay of the Public Health Laboratory Service, Perth, for phage typing the organisms.

\section{REFERENCES}

ANNEAR, D. I., AND GrubB, W. B. 1969. Pigmentation of medium by cultures of Staphylococcus aureus. Med.J. Austral., 2, 1107.

BARBER, MARY 1949. The incidence of penicillin-sensitive variant colonies in penicillinaseproducing strains of Staphylococcus pyogenes. J. Gen. Microbiol., 3, 274.

Evans, R. J., AND Waterworth, Pamela M. 1966. Naturally-occurring fusidic acid resistance in staphylococci and its linkage to other resistances. J. Clin. Path., 19, 555.

MaY, J. W., AND Houghton, R. H. 1965. A simple multipoint inoculator. Lab. Pract., 14, 168.

Mitsuhashi, S., Hashimoto, H., Kono, M., and Morimura, M. 1965. Drug resistance of staphylococci. II. Joint elimination and joint transduction of the determinants of penicillinase production and resistance to macrolide antibiotics. J. Bact., 89, 988.

Novick, R. P. 1967. Penicillinase plasmids of Staphylococcus aureus. Fedn Proc., 26, 29.

Novick, R. P., AND RichmoND, M. H. 1965. Nature and interactions of the genetic elements governing penicillinase synthesis in Staphylococcus aureus. J. Bact., 90, 467.

Novick, R. P., AND Roth, Christine 1968. Plasmid-linked resistance to inorganic salts in Staphylococcus aureus. J. Bact., 95, 1335.

RICHMOND, M. H. 1968. The plasmids of Staphylococcus aureus and their relation to other extrachromosomal elements in bacteria. Adv. Microbial Physiol., 2, 43.

RICHMOND, M. H., AND JOHN, MADELEINE 1964. Co-transduction by a staphylococcal phage of the genes responsible for penicillinase synthesis and resistance to mercury salts. Nature, Lond., 202, 1360. 